\title{
Manejo de ferrugem da soja com fungicidas em tratamento de sementes e aplicação foliar
}

\section{Management of soybean rust with fungicides for seed treatment and foliar application}

\author{
Fernando Godinho de Araújo ${ }^{1 *}$; Mara Rúbia da Rocha²; Renata Alves Aguiar ${ }^{1}$; \\ Riccely Ávila Garcia ${ }^{1}$; Marcos Gomes da Cunha ${ }^{2}$
}

\section{Resumo}

A soja é uma das principais culturas de exploração econômica no Brasil, mas tem seu potencial produtivo comprometido por uma série de doenças que afetam a cultura. $\mathrm{O}$ objetivo do presente trabalho foi avaliar a eficiência da incorporação de fluquinconazol ao tratamento de semente de soja, no manejo da ferrugem asiática (Phakopsora pachyrhizi), associado a diferentes fungicidas na parte aérea. $\mathrm{O}$ trabalho constou de dois ensaios dispostos lado a lado no campo. O primeiro ensaio constou de vários fungicidas empregados no controle de ferrugem asiática da soja (azoxistrobina, ciproconazol, trifloxistrobina, tebuconazol e protioconazol) e de tratamento das sementes com carbendazim + thiram e fluquinconazol. O segundo ensaio foi idêntico ao primeiro, mas sem a utilização de fluquinconazol. Esse experimento foi realizado em dois locais - Goiânia (GO) e Senador Canedo (GO). Em ambos os locais, os fungicidas aplicados via foliar foram eficientes no manejo da ferrugem da soja, mas a incorporação de fluquinconazol não aumentou significativamente o controle do fungo $P$. pachyrhizi, uma vez que não retardou o aparecimento da doença nem acarretou ganhos significativos de produtividade.

Palavras-chave: Glycine max, Phakopsora pachyrhizi, fluquinconazol

\begin{abstract}
Soybean is an important crop in the economical agriculture of Brazil and it has productive potential compromised by a variety of diseases affecting the crop such as soybean rust (Phakopsora pachyrhizi). The aim of this study was to evaluate the efficiency of incorporating fluquinconazole into the soybean seed treatment in the management of the disease, associated with spray of different fungicides on leaves. The work consisted of two trials side by side on the field. The first trial consisted of several fungicides used to control soybean rust (azoxistrobin, cyproconazole, trifloxystrobin, tebuconazole and prothioconazole) and seed treatment with carbendazim + thiram, and fluquinconazole. The second trail was identical to the first, but without the use of fluquinconazole. The experiment was carried out into two localities - Goiânia (GO) and Senador Canedo (GO). In both experiments, fungicides applied on leaves were effective controlling soybean rust, however the incorporation of fluquinconazole on the soybean seed treatment did not increase the control of the disease since neither it delayed the onset of the disease nor significantly increased the productivity of the crop.
\end{abstract}

Key words: Glycine max, Phakopsora pachyrhizi, fluquinconazole

1 Doutorando(a) do Programa de Pós-graduação em Agronomia, PPGA, Universidade Federal de Goiás, UFG. Goiânia, GO. E-mail: godinhoaraujo@hotmail.com; renatalvesufg@yahoo.com.br; riccelyavila@yahoo.com.br

2 Profs. da Escola de Agronomia e Engenharia de Alimentos, EA, UFG, Goiânia, GO. E-mail: mara.rocha@pq.cnpq.br; mgc@ agro.ufg.br

* Autor para correspondência 


\section{Introdução}

A soja é a mais importante oleaginosa cultivada no mundo, sendo o Brasil o segundo maior produtor mundial. A produção brasileira, até o inicio dos anos 80 , concentrou-se na região Centro-Sul do Brasil. A partir daí, a região Centro-Oeste teve um aumento significativo na participação da safra brasileira (TECNOLOGIAS, 2008). O complexo soja colheu aproximadamente 68,5 milhões de toneladas na safra 2009/10, com uma área plantada de 23,4 milhões de hectares e uma produtividade média de $2.900 \mathrm{~kg}$ (CONAB, 2010). A produtividade esperada para a cultura da soja ultrapassa os $4.000 \mathrm{~kg}$, no entanto, em função da grande gama de doenças que podem afetar a cultura, esse patamar quase sempre não é atingido.

No Brasil, as perdas anuais de produção causadas por ataque de fitopatógenos são estimadas em cerca de $15 \%$ a $20 \%$, entretanto, em determinadas condições, algumas doenças podem ocasionar perdas de quase 100\% (TECNOLOGIAS, 2010). Dentre essas doenças, a ferrugem (Phakopsora pachyrhizi Sidow) destaca-se como uma das mais ameaçadoras à cultura. É uma doença encontrada extensivamente em diversas regiões de produção de soja no mundo, onde perdas de até $90 \%$ são constatadas (BALARDIN, 2002). A doença é favorecida por chuvas bem distribuídas e longos períodos de molhamento superiores há 6 horas (MELO JÚNIOR; FERNANDES, 2010). A temperatura ótima para infecção varia entre $18^{\circ} \mathrm{e}$ $26,5^{\circ} \mathrm{C}$ (TECNOLOGIAS, 2008).

No Brasil, o manejo da ferrugem asiática inclui o uso de cultivares precoces e cultivares resistentes a $P$. pachyrhizi, a semeadura no início do período de plantio e a aplicação foliar de fungicidas. $O$ controle químico se configura como uma das principais medidas de controle, sendo os fungicidas pertencentes aos grupos estrobilutinas e triazóis, sozinhos ou em misturas, eficientes no controle da doença (GODOY; CANTERI, 2004). A partir da safra 2007/08, a utilização de misturas comerciais de triazóis e estrobirulina, tem sido recomendada em função da baixa eficiência dos triazóis (TECNOLOGIAS, 2010). Fungicidas com modo de ação específico possuem um maior risco de seleção de populações resistentes do patógeno, devendo dessa forma, alternar produtos com diferentes modos de ação ou utilizar misturas prontas de dois ou mais grupos para evitar a seleção de populações do fungo resistente a fungicidas (BEDIN et al., 2008). Desta forma, a presença de mais de um grupo de fungicidas com eficiência comprovada no controle de P. pachyrhizi é importante também no que diz respeito ao manejo de resistência de fungos a fungicidas.

A obtenção de uma população adequada de plantas é de fundamental importância para a rentabilidade econômica da cultura. O bom preparo do solo, a semeadura na época adequada, a utilização correta de agroquímiocos e a boa regulagem de semeadora são práticas essenciais, porém o seu sucesso está condicionado à utilização de sementes de boa qualidade. Quando a semeadura não é realizada em condições ideais o tratamento de sementes com fungicidas oferece uma garantia adicional ao estabelecimento da lavoura (HENNING, 2005).

O tratamento de sementes também tem sido utilizado como forma de manejo do fungo $P$. pachyrhizi. A utilização do fungicida fluquinconazol tem atrasado o início de epidemias, o progresso da ferrugem da soja e a desfolha das plantas (REZENDE; JULIATTI, 2010). No entanto, essa tecnologia não dispensa o tratamento padrão de sementes com fungicidas nem permite atrasar ou diminuir o número de aplicações de fungicidas foliares (TECNOLOGIAS, 2010).

O fluquinconazol é um fungicida sistêmico do grupo dos triazóis, registrado atualmente para as culturas do crisântemo, feijão, gladíolo, maça, melão, pêssego, soja e trigo (AGROFIT, 2010). Os triazóis agem inibindo a síntese de esteróis, denominados de azóis, sendo caracterizados por qualquer heterocíclico pentagonal insaturado, 
contendo átomos de carbono e pelo menos um átomo de hidrogênio, com ação protetora ou curativa contra fungos fitopatogênicos. Portanto, podem agir contra a germinação de esporos, a formação do tubo germinativo e no apressório; mesmo que haja a penetração do patógeno nos tecidos tratados, o produto atuará inibindo o haustório e/ ou crescimento micelial no interior dos tecidos (FORCELINI, 1994).

O presente trabalho teve como objetivo avaliar a eficiência da incorporação de fluquinconazol ao tratamento de semente de soja no manejo da ferrugem asiática, associado a diferentes fungicidas na parte aérea (azoxistrobina, ciproconazol, tiametoxan, trifloxistrobina, tebuconazol e protioconazol).

\section{Materiais e Métodos}

O trabalho constou de dois experimentos, um realizado no município de Goiânia e outro no município de Senador Canedo, em Goiás, nos meses de dezembro a abril de 2008. O delineamento experimental foi o de blocos ao acaso, com 6 tratamentos e 4 repetições. As parcelas foram constituídas de 6 linhas de soja com 7,0 m de comprimentos e espaçadas de $0,50 \mathrm{~m}$ entre linhas, totalizando $21,0 \mathrm{~m}^{2}$. A densidade de semeadura foi de 13 a 15 sementes/m linear de sulco de plantio.

Em cada localidade, instalou-se dois ensaios dispostos lado a lado no campo. O primeiro ensaio constou de vários programas de controle de ferrugem asiática da soja ( $P$. pachyrhizi) com aplicação foliar de fungicidas e de tratamento das sementes com Derosal Plus (carbendazim + thiram), na dose de $200 \mathrm{~mL} .100 \mathrm{~kg}^{-1}$ de sementes, e Atento (fluquinconazol), na dose de $300 \mathrm{~mL} .100$ $\mathrm{kg}^{-1}$ de sementes, conforme descrito na Tabelas $1 \mathrm{e}$ 2. O segundo ensaio foi idêntico ao primeiro, mas sem a utilização do tratamento de sementes com fluquinconazol.
A partir da primeira aplicação foliar de fungicidas, as avaliações de severidade da ferrugem foram feitas semanalmente até a testemunha atingir $80 \%$ de desfolha. A estimativa da severidade da ferrugem (\% de área foliar coberta por sintomas) foi feita com auxílio de escala diagramática (CANTERI; GODOY, 2003). Para isso foram coletados 20 trifólios nas linhas centrais de cada parcela, no terço médio e inferior das plantas. As amostras foliares foram avaliadas sob microscópio estereoscópico. A média desses valores foi considerada a severidade de doença na parcela. Os dados de severidade da parcela e os intervalos entre as avaliações foram usados para o cálculo da área abaixo da curva de progresso da doença (AACPD).

A produtividade e peso de mil grãos foram avaliados colhendo-se plantas em 5 metros nas 2 linhas centrais da parcela, totalizando $5,0 \mathrm{~m}^{2}$. Os valores obtidos foram convertidos para $\mathrm{kg} / \mathrm{ha}$ a $13 \%$ de umidade pela fórmula:

$$
\mathrm{Kg} / \mathrm{ha}=(100-\mathrm{US}) \mathrm{PP} /(100-13) \mathrm{AP} / 10
$$

\section{Onde:}

US = umidade da semente colhida

$\mathrm{PP}=$ peso da colheita de cada parcela

$\mathrm{AP}=$ área útil da parcela.

Os dados obtidos foram submetidos à análise de variância e, quando significativas, as médias comparadas pelo teste de Tukey a $5 \%$ de probabilidade.

Experimento Goiânia - Os ensaios foram conduzidos na área experimental da Escola de Agronomia e Engenharia de Alimentos da UFG, no município de Goiânia, Goiás. A adubação de plantio constou de $350 \mathrm{~kg} / \mathrm{ha}$ da fórmula 4-14-8, sendo a cultivar de soja BRS Valiosa RR semeada em 2 de dezembro de 2008. 
Tabela 1. Nome comercial, ingrediente ativo e químico dos produtos utilizados, Goiânia e Senador Canedo, GO, 2008/09.

\begin{tabular}{lcc}
\hline Nomes comerciais & Ingrediente Ativo & Grupo Químico \\
\hline Priori Xtra & Azoxistrobina + ciproconazol & Estrobilurina + Triazol \\
Adante & Tiametoxan + ciproconazol & Neonicotinóide + Triazol \\
Fox & Trifloxistrobina + protioconazol & Estrobilurina + Triazolinthione \\
Nativo & Trifloxistrobina + tebuconazol & Estrobilurina + Triazol \\
Nimbus & Óleo Mineral & Hidrocarbonetos alifáticos \\
Aureo & Éster metílico de óleo de soja & Éster metilado de óleo de soja \\
Atento & Fluquinconazol & Triazol \\
\hline
\end{tabular}

Fonte: AGROFIT, 2010.

Tabela 2. Tratamentos utilizados, dose do produto comercial e do ingrediente ativo, para o controle da ferrugem asiática (Phakopsora pachyrhizi) na cultura da soja (cv. BRS Valiosa RR) em Goiânia e Senador Canedo, GO, safra 2008/2009.

\begin{tabular}{lcc}
\hline Tratamento & $\begin{array}{c}\text { Dose produto comercial } \\
\left(\mathrm{g}, \mathrm{mL} \cdot \mathrm{ha}^{-1}\right)\end{array}$ & $\begin{array}{c}\text { Dose ingrediente ativo } \\
\left(\mathrm{g} \cdot \mathrm{ha}^{-1}\right)\end{array}$ \\
\hline Testemunha & --- & -- \\
Azoxistrobina + ciproconazol + óleo mineral & $300^{1}+600^{2}$ & $84+256,8$ \\
Azoxistrobina + ciproconazol + óleo mineral na 1a e 2a aplicação & $300+600$ & $84+256,8$ \\
Tiametoxan + ciproconazol + éster metílico de óleo de soja na & & \\
$3^{\mathrm{a}}$ aplicação & $150+600$ & $90+256,8$ \\
Trifloxistrobina + tebuconazol + éster metílico de óleo de soja & $500+500$ & $150+360$ \\
Trifloxistrobina + protioconazol + éster metílico de óleo de soja & $300+500$ & $97,5+720$ \\
Trifloxistrobina + protioconazol + éster metílico de óleo de soja & $300+500$ & $97,5+720$ \\
na 1 a aplicação & & \\
Trifloxistrobina + tebuconazol + éster metílico de óleo na 2a e & & $150+360$ \\
3 $^{\mathrm{a}}$ aplicação & $500+500$ & \\
\hline
\end{tabular}

${ }^{1}$ Dosagem do fungicida

${ }^{2}$ Dosagem do adjuvante

Fonte: Adaptado de AGROFIT, 2010.

As pulverizações foram iniciadas preventivamente, na fase R2 (florescimento pleno: maioria dos racemos com flores abertas). A primeira pulverização foi no dia 9 de fevereiro de 2009 , entre 9:00 e 10:00 horas. A temperatura média foi de $28^{\circ} \mathrm{C}$ e a UR de $76 \%$. A segunda aplicação, no dia 24 de fevereiro, entre 13:00 e 14:00 horas. A temperatura média foi de $30^{\circ} \mathrm{C}$ e a UR de $88 \%$. A terceira aplicação, foi dia 13 de março, entre 16:00 e 17:00 horas. A temperatura média foi de $30^{\circ} \mathrm{C}$ e a UR de $88 \%$. Todas as aplicações foram feitas com pulverizador costal pressurizado a $\mathrm{CO}_{2}$ com pressão constante de $80 \mathrm{lb} / \mathrm{pol}^{2}$, bico tipo leque 110.02. O volume de calda aplicada foi de $200 \mathrm{~L} / \mathrm{ha}$. A barra de aplicação possuía 3,0 m de largura e seis bicos, espaçados de 0,50 m de distância. A altura da barra de aplicação foi de aproximadamente $0,30 \mathrm{~m}$ a partir do topo das plantas.

Experimento Senador Canedo - Os ensaios foram realizados em área experimental do Centro Tecnológico para Pesquisas Agropecuárias (CTPA) no município de Senador Canedo - GO. A semeadura da cultivar de soja BRS Valiosa RR foi realizada em 13 de dezembro de 2008. No plantio foi feita adubação com a fórmula 4-14-8 (NPK), na dosagem de $300 \mathrm{~kg} \cdot \mathrm{ha}^{-1}$. 
Foi feito monitoramento de incidência da ferrugem semanalmente na área do ensaio tendo as pulverizações se iniciado quando as plantas atingiram o estádio $\mathrm{R} 1$, preventivamente à incidência da doença. As pulverizações foram realizadas entre 9:00 e 11:30h ou a partir das 16:00h. A primeira aplicação foi realizada no dia 09 de fevereiro de 2009, com temperatura de $30^{\circ}$ C e UR de $84 \%$. A segunda aplicação foi realizada no dia 24 de fevereiro, com temperatura de $28^{\circ} \mathrm{C}$ e UR de $83 \%$. A terceira aplicação foi no dia 13 de abril , com temperatura de $28^{\circ} \mathrm{C}$ e UR de $88 \%$. Os equipamentos e condições de aplicação foram idênticos aos do experimento de Goiânia, exceto o volume de calda aplicada que foi $150 \mathrm{~L} / \mathrm{ha}$.

\section{Resultados e Discussão}

A área abaixo da curva de progresso da doença (AACPD) da testemunha nos experimentos de Goiânia e de Senador Canedo foi estatisticamente menor que as AACPD dos demais tratamentos tanto para os ensaios com ou sem fluquinconazol (Tabelas 3 e 4). Esses resultados indicam que todos os programas de manejo do fungo $P$. pachyrhizi com fungicidas foram eficientes no controle da doença independente do tratamento de sementes adicional com fluiquinconazol. A eficiência dos fungicidas a base de azoxistrobina, ciproconazol, trifloxistrobina, tebuconazol e protioconazol no manejo da ferrugem asiática foi comprovada, sendo esses resultados condizentes com os resultados obtidos em outras regiões do país (GODOY; CANTERI, 2004; SOARES et al., 2004; NAVARINI et al., 2007; GODOY; HENNING, 2008).

No experimento de Goiânia, foi possível detectar diferenças significativas dentro de todos os tratamentos com relação à utilização de fluquinconazol, exceto para o tratamento tiametoxan + ciproconazol ( $3^{\text {a }}$ ap.) (Tabela 3$)$. O fluquinconazol, nesse caso, sempre esteve associado a uma menor AACPD. Já no experimento realizado no município de Senador Canedo, a influência do fluquinconazol não foi observada da mesma forma que no experimento de Goiânia. O tratamento adicional de sementes chegou a aumentar a AACPD na testemunha e nos programas de pulverização com azoxistrobina + ciproconazol ou trifloxistrobina + tebuconazol (Tabela 4). Rezende e Juliatti (2010) observaram resultados semelhantes aos obtidos no presente trabalho, no qual as avaliações da AACPD não diferiram entre os tratamentos com e sem a utilização de fluquinconazol, mas os tratamentos diferiram da testemunha. Esses resultados mostram que os fungicidas testados, já registrados para a cultura da soja, pertencentes aos grupos estrobilurinas e triazóis, sozinhos e em misturas, são eficientes para reduzir a severidade do ataque de $P$. pachyrhizi, independente do tratamento de sementes com fluquinconazol (GODOY; CANTERI, 2004).

O horário de aplicação é um componente de grande importância. As aplicações realizadas às 12:00h são expostas a condições climáticas que favoreceram a ocorrência de perdas por deriva e evaporação das gotas pulverizadas. Nessa condição a cobertura proporcionada no alvo de aplicação é inferior às demais condições, acarretando em menor eficácia (BONINI, 2003; MENEGHETTI, 2006). Ao comparar as AACPD nos experimentos realizados, é possível notar que, de modo geral, as AACPD do experimento conduzido em Goiânia foram maiores que as do trabalho conduzido em Senador Canedo. Esse fato pode ter ocorrido em função do horário de aplicação dos fungicidas para o controle da ferrugem asiática. A segunda aplicação, aos 24 dias do mês de fevereiro de 2009, foi realizada entre 13:00 e 14:00 horas. Esse horário não é recomendado em função da predisposição de ocorrência de deriva. Em condições de vento acentuado, as gotas pequenas produzidas pelas pontas dos bicos de pulverização apresentam maior risco de perdas por deriva e, conseqüentemente, menor deposição e menor eficiência no controle da doença (CUNHA et al., 2008).

Quando o programa de pulverização não expressa o seu maior potencial é possível visualizar o efeito 
do fluquinconazol. Esse triazol de ação sistêmica age inibindo o haustório e/ou crescimento micelial no interior dos tecidos (FROCELINI, 1994). Dessa forma, plantas oriundas de sementes tratadas com fluquinconazol, em áreas com programa de pulverização deficitário, têm uma maior capacidade de resistir ao avanço da infecção do fungo $P$. pachyhrizi.

A desfolha, em ambos os experimentos, apresentou diferenças significativas entre a testemunha e os programas de pulverização, exceto para o experimento no município de Goiânia sem a utilização do tratamento adicional de sementes com fluquinconazol (Tabelas 3 e 4). Nesse experimento, a desfolha da testemunha se assemelhou a desfolha dos tratamentos azoxistrobina + ciproconazol; azoxistrobina + ciproconazol $\left(1^{\mathrm{a}}\right.$ e $2^{\mathrm{a}}$ ap. $)$ e tiametoxan + ciproconazol ( $3^{\mathrm{a}}$ ap. $)$; trifloxistrobina + tebuconazol. Quando o tratamento adicional de sementes com fluquinconazol foi utilizado, a desfolha de todos os programas de manejo é significativamente menor que a desfolha da testemunha (Tabela 3). No experimento de Goiânia, a utilização ou não de fluquinconazol afetou significativamente a desfolha de todos os tratamentos exceto da testemunha e do programa de pulverização trifloxistrobina + protioconazol $\left(1^{\mathrm{a}} \mathrm{e}\right.$ $2^{\mathrm{a}}$ ap.) e trifloxistrobina + tebuconazol ( $3^{\mathrm{a}}$ ap.). No tocante a desfolha no experimento realizado em Senador Canedo, não houve diferença significativa nos índices de desfolha em função do uso ou não de fluquinconazol (Tabela 4).
A produtividade teve uma grande variação nos experimentos conduzidos. Para o experimento realizado no município de Senador Canedo, todos os tratamentos diferiram da testemunha exceto o tratamento trifloxistrobina + tebuconazol, sem o tratamento adicional de sementes com fluquinconazol (Tabela 4). Esse resultado já poderia ser previsto ao se analisar a AACPD, pois esse tratamento foi o que teve maior área quando comparado com os demais fungicidas utilizados. Esses resultados são semelhantes aos obtidos por Rezende e Juliatti (2010). Nesse experimento também não houve diferença significativa com relação à utilização do tratamento de sementes com flunquiconazol.

O experimento de Goiânia, quando não foi realizadootratamentoadicionaldesementes, somente o tratamento trifloxistrobina + protioconazol diferiu da testemunha. Quando foi realizado o tratamento adicional com fluquinconazol, essa diferença não perdurou. Foram identificadas diferenças entre a testemunha e os tratamentos azoxistrobina + ciproconazol $\left(1^{\mathrm{a}} \mathrm{e} 2^{\mathrm{a}}\right.$ ap. $)$ e tiametoxan + ciproconazol $\left(3^{\text {a }}\right.$ ap. $)$, e trifloxistrobina + tebuconazol. A baixa produtividade do experimento realizado em Goiânia se deve em grande parte a baixa eficiência no manejo da doença. Condições adversas no momento da aplicação de fungicidas foliares podem resultar em uma maior AACPD, consequentemente maior desfolha e menor produtividade. 
Tabela 3. Área abaixo da curva de progresso da doença (AACPD), desfolha e produtividade dos ensaios realizados no município de Goiânia, GO, com e sem a utilização de tratamento adicional de sementes com fluquinconazol, na safra 2008/09.

\begin{tabular}{|c|c|c|c|c|c|c|}
\hline \multirow{2}{*}{ Tratamentos } & \multicolumn{2}{|c|}{ AACPD } & \multicolumn{2}{|c|}{ Desfolha } & \multicolumn{2}{|c|}{ Produtividade } \\
\hline & $\mathbf{S}^{1}$ & $\mathrm{C}^{2}$ & $\mathbf{S}$ & $\mathrm{C}$ & $\mathbf{S}$ & $\mathrm{C}$ \\
\hline Testemunha & 692,61 a A & 626,79 a B & $88,75 \mathrm{a} \quad \mathrm{A}$ & $81,25 \mathrm{a} A$ & $1994,76 \mathrm{a} \quad \mathrm{A}$ & $2135,96 \mathrm{a} \quad \mathrm{A}$ \\
\hline Azoxistrobina + ciproconazol & $212,60 \mathrm{~b} \mathrm{~A}$ & $163,48 \mathrm{~b} \mathrm{~B}$ & $68,75 \mathrm{ab} A$ & $43,75 b$ B & $2380,47 \mathrm{ab} \mathrm{B}$ & $2711,68 b \quad A$ \\
\hline $\begin{array}{l}\text { Azoxistrobina }+ \text { ciproconazol }\left(1^{\mathrm{a}} \text { e } 2^{\mathrm{a}} \text { ap. }\right) \\
\text { Tiametoxan }+ \text { ciproconazol }\left(3^{\mathrm{a}} \text { ap. }\right)^{3}\end{array}$ & $213,60 \mathrm{~b} \mathrm{~A}$ & $177,33 \mathrm{~b} \mathrm{~A}$ & $68,75 \mathrm{ab} A$ & $42,50 \mathrm{~b} \mathrm{~B}$ & $2486,06 \mathrm{ab} A$ & $2701,50 \mathrm{~b} \quad \mathrm{~A}$ \\
\hline Trifloxistrobina + tebuconazol & $240,89 \mathrm{~b} \mathrm{~A}$ & $186,78 \mathrm{~b} \mathrm{~B}$ & $67,50 \mathrm{ab} A$ & $46,25 b \mathrm{~B}$ & $2387,03 \mathrm{ab} \mathrm{A}$ & $2541,05 \mathrm{ab} \mathrm{A}$ \\
\hline Trifloxistrobina + protioconazol & $210,94 \mathrm{~b} \mathrm{~A}$ & $165,73 \mathrm{~b} \mathrm{~B}$ & $57,50 \mathrm{~b} \quad \mathrm{~A}$ & $38,75 b \mathrm{~B}$ & $2410,84 b \quad \mathrm{~A}$ & $2483,64 \mathrm{ab} A$ \\
\hline $\begin{array}{l}\text { Trifloxistrobina }+ \text { protioconazol }\left(1^{\mathrm{a}} \text { e } 2^{\mathrm{a}} \text { ap. }\right) \\
\text { Trifloxistrobina }+ \text { tebuconazol }\left(3^{\mathrm{a}} \text { ap. }\right)^{4}\end{array}$ & 244,17 b A & $196,57 \mathrm{~b} \mathrm{~B}$ & $56,25 \mathrm{~b} \quad \mathrm{~A}$ & $45,00 \mathrm{~b} \mathrm{~A}$ & $2399,20 \mathrm{ab} \mathrm{A}$ & $2551,33 \mathrm{ab} A$ \\
\hline
\end{tabular}

${ }^{1} \mathrm{~S}$ : sem tratamento adicional de sementes com fluquinconazol

${ }^{2} \mathrm{C}$ : com tratamento adicional de sementes com fluquinconazol

${ }^{3}$ Utilização de azoxistrobina + ciproconazol na primeira e segunda aplicação foliar de fungicida e de tiametoxan + ciproconazol na terceira aplicação. ${ }^{4}$ Utilização de trifloxistrobina + protioconazol na primeira e segunda aplicação foliar de fungicida e de trifloxistrobina + tebuconazol na terceira aplicação.

Médias seguidas pelas mesmas letras minúsculas nas colunas e maiúsculas nas linhas não diferem entre si, pelo Teste de Tukey ( $<<0,05$ ).

Fonte: Elaboração dos autores.

Tabela 4. Área abaixo da curva de progresso da doença (AACPD), desfolha e produtividade dos ensaios realizados no município de Senador Canedo, GO, com e sem a utilização de tratamento adicional de sementes com fluquinconazol, na safra 2008/09.

\begin{tabular}{|c|c|c|c|c|c|c|}
\hline \multirow{2}{*}{ Tratamentos } & \multicolumn{2}{|c|}{ AACPD } & \multicolumn{2}{|c|}{ Desfolha } & \multicolumn{2}{|c|}{ Produtividade } \\
\hline & $\mathbf{S}^{1}$ & $\mathbf{C}^{2}$ & $\mathbf{S}$ & $\mathbf{C}$ & $\mathbf{S}$ & $\mathrm{C}$ \\
\hline Testemunha & $633,93 a \mathrm{~B}$ & $770,49 a \quad A$ & 87,50 a & 86,25 a & 2598,29 a & $2296,19 \mathrm{a}$ \\
\hline Azoxistrobina + ciproconazol & $82,30 \mathrm{bcB}$ & $137,38 \mathrm{c}$ A & $42,50 \mathrm{~b}$ & $42,50 \mathrm{~b}$ & $4291,62 \mathrm{~b}$ & $4072,08 \mathrm{~b}$ \\
\hline $\begin{array}{l}\text { Azoxistrobina }+\operatorname{ciproconazol}\left(1^{\mathrm{a}} \text { e } 2^{\mathrm{a}} \text { ap. }\right) \\
\text { Tiametoxan }+ \text { ciproconazol }\left(3^{\mathrm{a}} \text { ap. }\right)^{3}\end{array}$ & $79,71 \mathrm{c} \mathrm{A}$ & $75,45 \mathrm{~cd} \mathrm{~A}$ & $47,50 \mathrm{~b}$ & $47,50 \mathrm{~b}$ & $4239,56 \mathrm{~b}$ & $4421,32 \mathrm{~b}$ \\
\hline Trifloxistrobina + tebuconazol & $150,59 \mathrm{~b}$ B & $230,26 \mathrm{~b} A$ & $47,50 \mathrm{~b}$ & $46,25 \mathrm{~b}$ & $3950,01 \mathrm{ab}$ & $4004,32 \mathrm{~b}$ \\
\hline Trifloxistrobina + protioconazol & $18,55 \mathrm{c} \mathrm{A}$ & $24,70 \mathrm{~d} A$ & $42,50 \mathrm{~b}$ & $42,50 \mathrm{~b}$ & $4484,89 \mathrm{~b}$ & $4614,52 \mathrm{~b}$ \\
\hline $\begin{array}{l}\text { Trifloxistrobina }+ \text { protioconazol }\left(1^{\mathrm{a}} \text { e } 2^{\mathrm{a}} \text { ap. }\right) \\
\text { Trifloxistrobina }+ \text { tebuconazol }\left(3^{\mathrm{a}} \text { ap. }\right)^{4}\end{array}$ & $58,35 \mathrm{c} \mathrm{A}$ & $65,27 \mathrm{~d} A$ & $45,00 \mathrm{~b}$ & $45,00 \mathrm{~b}$ & $4853,76 \mathrm{~b}$ & $4148,83 \mathrm{~b}$ \\
\hline
\end{tabular}

${ }^{1} \mathrm{~S}$ : sem tratamento adicional de sementes com fluquinconazol

${ }^{2} \mathrm{C}$ : com tratamento adicional de sementes com fluquinconazol

${ }^{3}$ Utilização de azoxistrobina + ciproconazol na primeira e segunda aplicação foliar de fungicida e de tiametoxan + ciproconazol na terceira aplicação. ${ }^{4}$ Utilização de trifloxistrobina + protioconazol na primeira e segunda aplicação foliar de fungicida e de trifloxistrobina + tebuconazol na terceira aplicação.

Médias seguidas pelas mesmas letras minúsculas nas colunas e maiúsculas nas linhas não diferem entre si, pelo Teste de Tukey ( $<<0,05$ ).

Fonte: Elaboração dos autores. 
O tratamento de semente com fluquinconazol não mostrou atraso na evolução da doença, conforme indicado em (TECNOLOGIAS, 2010). Quando se tem problemas na aplicação de fungicidas foliares para o controle do P. pachyrhizi o tratamento adicional com fluquinconazol resulta em uma menor desfolha. No entanto esse comportamento não é verificado no tocante a produtividade. A utilização do tratamento adicional de sementes com fluquinconazol não resulta em incrementos de produtividade. Godoy e Henning (2008) também concluíram que o tratamento de semente de soja com fluquinconazol não atrasa o aparecimento dos primeiros sintomas nem a evolução da doença e não apresenta benefício no manejo da ferrugem.

Os programas de pulverizações testados para o controle do fungo P. pachyrhizi são eficientes. A utilização do tratamento de sementes adicional com fluquinconazol não proporciona aumento de produtividade à cultura da soja, no entanto, diminui a AACPD e a desfolha quando se tem falhas no programa de pulverizações contra a ferrugem asiática.

\section{Referências}

AGROFIT, M. D. A. P. E. A. Sistema de agrotóxicos fitossanitários. Brasília: Ministério da Agricultura Pecuária e Abastecimento, 2010.

BALARDIN, R. S. Doenças da soja. Santa Maria: Universidade Federal de Santa Maria, 2002.

BEDIN, C.; MENDES, L. B.; TRECENTE, V. C.; LOPES, R. L. B.; BOSQUE, G. G. Controle da ferrugem asiática na cultura da soja. Revista Científica Eletrônica de Agronomia, Garça, v. 7, n. 13, p. 1-6, 2008.

BONINI, J. V. Tecnologia de aplicação de fungicidas na cultura da soja. 2003. Tese (Mestrado em Agronomia) Universidade Federal de Santa Maria, Santa Maria.

CANTERI, M. G.; GODOY, C. V. Escala diagramática da ferrugem da soja ( $P$. pachyrhizi). Summa Phytopathologica, Botucatu, v. 1, n. 1, p. 32, 2003.

COMPANHIA NACIONAL DE ABASTECIMENTO - CONAB. Séries históricas soja - Brasil. Brasília: CONAB, 2010.

CUNHA, J. P. A. R. D.; MOURA, E. A. C.; JÚNIOR, J. L. D. S.; ZAGO, F. A.; JULIATTI, F. C. Efeito de pontas de pulverização no controle químico da ferrugem da soja. Engenharia Agrícola, Jaboticabal, v. 28, n. 2, p. 283-291, 2008.

TECNOLOGIAS de produção de soja. Região Central do Brasil 2008. Londrina: Embrapa Soja: Embrapa Cerrados: Embrapa Agropecuária Oeste, 2008. (Sistemas de Produção. Embrapa Soja, n. 12).

Região Central do Brasil 2011. Londrina:

Embrapa Soja: Embrapa Cerrados: Embrapa Agropecuária Oeste, 2010. (Sistemas de Produção. Embrapa Soja, n. 14).

FORCELINI, C. A. Fungicidas inibidores da síntese de esteróis. I. Triazoles. Revisão Anual de Patologia de Plantas, Passo Fundo, v. 2, n. 1, p. 335-351, 1994.

GODOY, C. V.; CANTERI, M. G. Efeitos protetor, curativo e erradicante de fungicidas no controle da ferrugem da soja causada por Phakopsora pachyrhizi, em casa de vegetação. Fitopatologia Brasileira, Brasília, v. 29, n. 1, p. 97-101, 2004.

GODOY, C. V.; HENNING, A. A. Tratamento de semente e aplicação foliar de fungicidas para controle de ferrugem-da-soja. Pesquisa Agropecuária Brasileira, Brasília, v. 43, n. 10, p. 1297-1302, 2008.

HENNING, A. A. Patologia e tratamento de sementes: noções gerais. 2. ed. Londrina: Embrapa Soja, 2005.

MELO JÚNIOR, H. B. D.; FERNANDES, J. J. Período de molhamento foliar para ocorrência de ferrugem asiática (Phakopsora pachyrhizi) em dois cultivares de soja (Glycine max). Enciclopédia Biosfera, Goiânia, v. 6, n. 11, p. 1-11, 2010.

MENEGHETTI, R. C. Tecnologia de aplicação de fungicidas na cultura do trigo. 2006. Tese (Mestrado em Engenharia Agrícola) - Universidade Federal de Santa Maria, Santa Maria.

NAVARINI, L.; DALLAGNOL, L. J.; BALARDIN, R. S.; MOREIRA, M. T.; MENEGHETTI, R. C.; MADALOSSO, M. G. Controle químico da ferrugem asiática (Phakopsora pachyrhizi Sidow) na cultura da soja. Summa Phytopathologica, Botucatu, v. 33, n. 2, p. 182-186, 2007.

REZENDE, A. A.; JULIATTI, F. C. Tratamento de sementes de soja com fluquinconazole no controle da ferrugem asiática. Bioscience Journal, Uberlândia, v. 26, n. 1, p. 84-94, 2010.

SOARES, R. M.; RUBIN, S. D. A. L.; WIELEWICKI, A. P.; OZELAME, J. G. Fungicidas no controle da ferrugem asiática (Phakopsora pachyrhizi) a produtividade da soja. Ciência Rural, Santa Maria, v. 34, n. 4, p. 1245 1247, 2004. 